\title{
Diagnosis and subtype analysis of Blastocystis sp. in 442 patients in a hospital setting in the Netherlands
}

Aldert Bart $^{1 *}$, Ellen MS Wentink-Bonnema ${ }^{1}$, Henk Gilis $^{1}$, Nienke Verhaarr ${ }^{1}$, Carla JA Wassenaar ${ }^{1}$, Michèle van Vugt ${ }^{2}$, Abraham Goorhuis ${ }^{2}$ and Tom van Gool ${ }^{1,3}$

\begin{abstract}
Background: Blastocystis sp. are among the most commonly observed intestinal parasites in routine clinical parasitology. Blastocystis in humans consists of at least 9 genetic subtypes. Different subtypes of Blastocystis may be associated with differences in pathogenicity and symptomatology.

Methods: Advanced microscopy on two samples and sequence-confirmed PCR on a third sample from the same individual were used for Blastocystis diagnosis and subtype analyses on routine clinical samples in a university hospital.

Results: With a combined gold standard of sequence-confirmed PCR and positive advanced microscopy, 107 out of 442 (24.2\%) patients were diagnosed with Blastocystis. infection, which is a high frequency of detection in comparison to previous reports from industrialized countries. The sensitivity of microscopy and sequence-confirmed PCR was 99.1\% (106/107) and 96.3\% (103/107), respectively.

Among 103 typable samples, subtype 3 was most abundant $(n=43,42 \%)$, followed by subtypes 1 and 2 (both $n=23,22 \%)$, subtype $4(n=12,12 \%)$, and single samples with subtypes $6(1 \%)$ and subtype $7(1 \%)$. The prevalence of Blastocystis infection was 38\% in patients from the Department of Tropical Medicine and $18 \%$ in patients from other departments.

Conclusions: A high prevalence of Blastocystis infection was found with both advanced microscopy and sequenceconfirmed PCR in our patient population. Most cases were caused by subtypes ST1, ST2, ST3 and ST4. A significantly higher prevalence was found among patients with a history of recent travel to tropical countries.
\end{abstract}

Keywords: Blastocystis, Diagnosis, Microscopy, PCR, Molecular epidemiology

\section{Background}

Blastocystis is one of the most prevalent unicellular parasites in human faecal specimens. The prevalence of Blastocystis has been reported to range from $60 \%$ in tropical, subtropical and developing countries [1] to as low as $0.5 \%$ in Japan [2]. Considerable variation in reported prevalence can be related to the epidemiological setting, especially the patient population studied, but is probably also due to differences in the diagnostic

\footnotetext{
* Correspondence: a.bart@amc.uva.nl

'Parasitology Section, Department of Medical Microbiology, Center for Infection and Immunity Amsterdam (CINIMA), Academic Medical Center, Amsterdam, The Netherlands

Full list of author information is available at the end of the article
}

methods used [3]. Transmission of Blastocystis is suggested to be by the faecal-oral route, e.g. via contaminated water or food, with sources being both humans and animals [4-6].

Clinical features of Blastocystis infection are reported to include nausea, anorexia, abdominal pain, flatulence and acute or chronic diarrhoea $[7,8]$. In addition, an association is suggested with symptomatology in irritable bowel syndrome [9-13] and, as an opportunistic pathogen, in immunocompromised patients $[14,15]$. Other studies however suggest Blastocystis to be a commensal parasite in humans without any pathogenic role [16-18].

Conflicting views about pathogenicity may be related to the number of infecting parasites present, duration of

\section{Ciomed Central}


infection (acute or chronic), host genetic factors or to different subtypes or species of Blastocystis infecting humans [3,19-27]. Currently 17 subtypes or species of Blastocystis have been described based on sequence analysis, of which 9 have been reported in humans [28-31].

For proper study of prevalence, epidemiology and pathogenic potential of Blastocystis, reliable and practical diagnosis is of paramount importance. Techniques currently in use include microscopy, molecular detection, and xenic in vitro culture [3]. Recent studies suggest certain molecular methods to have highest sensitivities [26,32-34]. In this report we compare sequence-confirmed PCR to advanced microscopy, and describe the prevalence of Blastocystis infection and subtype determination in a large series of patients in The Netherlands.

\section{Methods}

\section{Samples and parasitological examination}

Stool samples of 442 patients submitted for routine parasitological examination at the Department of Clinical Parasitology, Academic Medical Center (AMC) were collected and processed with the Triple Faeces Test (TFT) as described previously [35]. Microscopy with the TFT has been part of routine clinical care in the AMC for 18 years. In brief, with the TFT every patient fills three tubes with stools on 3 consecutive days. Tubes of day 1 and day 3 are prefilled with SAF (sodium acetate-acetic acid-formalin), while the tube of day two is empty. After 3 days the complete TFT set is sent to the laboratory by the patient. Compliance of patients for proper delivery of the whole set is high [35]. SAF-stool suspensions of days 1 and 3 are studied in the laboratory by an iodine stained wet smear and, in case of undetermined protozoa, by a permanent Chlorazol Black stain [35]. Fresh (non-fixed) stool of day 2 is routinely processed by the formol ether concentration technique (FECT) for diagnosis of (cysts and eggs of) protozoa and helminths. In addition, on sample 2 auramine stain for Cryptosporidium, Uvitex 2B for Microsporidia, antigen tests and PCR are performed when indicated. In this study PCR for Blastocystis was performed on sample 2 .

For microscopic diagnosis of Blastocystis from each SAF sample approximately $10 \mu \mathrm{l}$ of SAF stool sample was mixed with approximately $20 \mu \mathrm{l}$ of Lugol's iodine solution and covered with a $21 \times 26 \mathrm{~mm}$ cover slip. The slide was examined for intestinal parasites, including Blastocystis for 300 optical fields with a magnification of $250 \times$ ( $20 \times$ objective and 12.5 eye piece) and, in case of suspected organisms, $500 \times(40 \times 12.5 \times)$. Duration of this examination per slide is 5 minutes on average. The diagnostic criterion for positivity of Blastocystis species was at least 2 clear vacuolar forms of the parasite in either of the two SAF samples. Granular or amoeboid stages of Blastocystis species were not studied.
Permanent staining with Chlorazol Black was routinely performed on any SAF sample where further determination was needed, as is true for Dientamoeba fragilis and other vegetative stages of protozoa. Results of iodine wet smear and Chlorazol Black stain were compared on a selected series of positive and negative samples. Technicians performing microscopic diagnosis had extensive experience in parasitological diagnosis and were blinded for the results of PCR.

\section{Molecular methods}

All non-fixed stools collected on the second day were mixed with Stool Transport and Recovery (S.T.A.R.) Buffer (Roche Applied Science) to an approximately 1:3 $\mathrm{v}: \mathrm{v}$ suspension and vortexed and stored for DNA isolation and PCR. DNA was isolated from all faecal suspensions using the MagNA Pure LC Isolation instrument for nucleic acid extraction (Roche Applied Science). To monitor the process of extraction and amplification, a universal control consisting of phocine herpesvirus type 1 (PhHV-1) was added to the clinical specimens [36]. Briefly, $20 \mu \mathrm{l}$ of faecal suspension was added to $500 \mu \mathrm{l}$ lysis buffer with 2500 copies PhHV-1 and prelysed for 10 minutes at room temperature. After centrifugation, $500 \mu \mathrm{l}$ of the lysate supernatant was used as input for the Total Nucleic Acid Isolation Kit (Roche Applied Science) as described by the manufacturer. Elution of nucleic acid was in a final volume of $50 \mu \mathrm{l}$. Blastocystis PCR was performed using primers and conditions described by Stensvold [33], aimed at a region of the small subunit rRNA gene of Blastocystis, using $15 \mu \mathrm{l}$ of isolated DNA as input in a total volume of $50 \mu \mathrm{l}$. Fifteen microliters of PCR product were size-separated by agarose gel electrophoresis and visualized by UV illumination after ethidium bromide staining. Diluted amplicons were sequenced on both strands using amplification primers with BigDyeTerminator chemistry (Applied Biosystems) and analysed on an ABI 3900 sequencer. Subtypes following the nomenclature proposed by Stensvold et al [21] were determined by comparison of obtained sequences to Genbank reference sequences using CodonCode Aligner program (CodonCode Corporation), and MEGA 4.0 [37]. For sequences that seemed to contain a mixed signal of multiple subtypes, the predominant subtype was assigned. Only samples for which a Blastocystis sequence was obtained were considered PCR and sequencing positive. Samples for which no Blastocystis sequence was obtained or for which there was no PCR amplicon while the PhHV1 control DNA was detected using real-time PCR [36], were considered negative. PCR and sequence interpretation were performed blinded to the microscopy results. Sequences were deposited in Genbank with accession numbers KF241991-KF242090. 


\section{Database and statistics}

Samples and patient data (sex, age, referring department) were anonymized and aggregated in a database. For evaluation of diagnostic tests using anonymized data no medical-ethical approval is required in The Netherlands, provided that patients declare no objection to such use of their samples. Computations of $P$ values using Pearson's $X^{2}$ test were performed at VassarStats website (http://vassarstats.net/index.html). $P$ values below or equal to 0.05 were regarded as significant.

\section{Results}

\section{Study population and microscopy}

From October 2008 to January 2009, 442 TFT sets from 442 patients were included in the study. The 442 patients comprised 230 females (median age 29, range 1-82) and 212 males (median age 34, range 0-87 years). Patients were referred by the Tropical Diseases Department (133), Emma Children's Hospital (103), general practicioners (70), Department of Internal Medicine (63), Department of Gastroenterology and Hepatology (45), or other departments (28).

In 148 out of 442 (33\%) patients one or more intestinal parasitic species were observed. Protozoa were observed in 148 out of 442 (33\%) and helminths in 3 out of $442(0.7 \%)$ patients.

Blastocystis were recognized in either of the two SAF preserved samples by microscopy of iodine stained wet smears in 106 out of $442(24.0 \%)$ patients in any of two samples. In 90 patients, both SAF samples (day 1 and day 3) were positive for Blastocystis. In 10 patients Blastocystis was observed only in the sample of the first day, and in 6 only in the sample of the third day. From 106 patients positive for Blastocystis with iodine wet smear, 34 times a permanent Chlorazol Black stain was performed for determination of other protozoa. In all cases Blastocystis were also observed with the Chlorazol Black stain. From 336 patients negative for Blastocystis with iodine wet smear, 81 times a permanent stained slide also was studied. In none of these Blastocystis was observed.

\section{PCR and Blastocystis subtype determination}

In 103 out of 442 (23.3\%) patients, sequence-confirmed PCR was positive. Six subtypes were observed among the 103 typable samples: subtype 3 was most abundant $(\mathrm{n}=43,41 \%)$, followed by subtypes 1 and 2 (both $\mathrm{n}=23$, $22 \%)$, subtype $4(\mathrm{n}=12,12 \%)$, and single samples with subtypes $6(1 \%)$ and subtype 7 (1\%) (Table 1).

\section{Gold standard, congruence between microscopy and PCR}

Assuming 100\% specificity of both microscopy and sequence-confirmed PCR, positive findings of both tests were combined to serve as a gold standard. Using this standard, 107 of 442 patients (24.2\%) were positive for Blastocystis. Microscopy, with reading of two SAF samples studied per patient, had a sensitivity of $99.1 \%(106 / 107)$. Sequence-confirmed PCR, performed on stool of the day 2 sample only, had a sensitivity of $96.3 \%$ (103/107).

In five samples, microscopy and sequence-confirmed PCR were not in agreement. This concerned 4 times a microscopy positive and sequence-PCR negative result, and one sequence- PCR positive and microscopy negative result. All five samples were retested with both methods. The four microscopically positive samples that were PCR and sequence negative, were all positive for the PhHV-1 extraction and amplification control, suggesting that PCR was not negative due to insufficient DNA extraction or inhibitory factors in the eluate, but due to the amount of Blastocystis DNA being below detection level. This is in accordance with the observation of only few parasites ( $<5$ per total slide) in two of these samples by microscopy. In the other two samples, more Blastocystis cells were observed. By agarose gel electrophoresis of PCR products, faint bands were visible in these samples. Apparently, the yield of PCR amplicon was insufficient to obtain good sequences in these two samples. Alternatively, the microscopical observations may be explained by structures that are morphologically highly similar to Blastocystis. In the one sample that was PCR and sequencing positive, but negative in microscopy, a weak band was observed in PCR that yielded a sequence. Possibly, only Blastocystis DNA or cysts were present in this sample, rather than intact Blastocystis cells.

Table 1 Blastocystis infection status of patients admitted via the Department of Tropical Medicine and patients admitted via other departments

\begin{tabular}{|c|c|c|c|c|c|c|c|c|}
\hline Patient samples & $\begin{array}{l}\text { Total patients } \\
\text { examined }\end{array}$ & $\begin{array}{l}\text { Blastocystis } \\
\text { positive (\%) }\end{array}$ & ST1 & ST2 & ST3 & ST4 & Other ST & $N S T^{a}$ \\
\hline Submitted via dept tropical diseases & 133 & $50(38 \%)^{b}$ & $13(26 \%)$ & $10(20 \%)^{c}$ & $20(40 \%)$ & $5(10 \%)$ & $1(2 \%)$ & $1(2 \%)$ \\
\hline Submitted via other departments & 309 & $57(18 \%)^{b}$ & $10(18 \%)$ & $13(23 \%)$ & $23(40 \%)$ & $7(12 \%)$ & $1(2 \%)$ & $3(5 \%)$ \\
\hline Total population & 442 & 107 (31\%) & $23(21 \%)$ & $23(21 \%)$ & $43(40 \%)$ & $12(11 \%)$ & $2(2 \%)$ & $4(4 \%)$ \\
\hline
\end{tabular}

aST: not subtypable. These isolates were positive by microscopy only.

${ }^{b} \mathrm{p}<0.0001$.

c one of these patients was negative by microscopy. 


\section{Relation between Blastocystis infection, subtype and patient characteristics}

In the patient population from the Department of Tropical Medicine, significantly ( $\mathrm{p}<0.0001$; Pearson's $\chi^{2}$ test) more Blastocystis infection was found $(38 \%, 50 / 133)$ as compared to patients from other departments (18\%, 57/ 309), as shown in Table 1 . There was no significant difference in the subtype distribution between patients presenting at the Department of Tropical Medicine and patients presenting at other departments.

No significant correlation between patient gender and Blastocystis infection was found. Patients with Blastocystis infection were more frequently coinfected with Dientamoeba fragilis $(17 \%, 18 / 107)$ than patients without Blastocystis infection $(8 \%, 26 / 335)$ ( $\mathrm{p}<0.006$; Pearson's $\chi^{2}$ test). No significant correlation of the subtype of Blastocystis and Dientamoeba infection was observed.

\section{Discussion}

In our hospital-derived study population, 24\% (107/ 442) of patients were infected with Blastocystis. This is comparable to the reported $23 \%$ prevalence found in a smaller ( $\mathrm{n}=93$ patients) hospital population in Denmark with direct PCR on stool samples [33], and higher than 19\% reported in Australia where a combination of microscopy, culture and PCR was used for diagnosis [32]. In similar teaching hospital settings in Italy [38] and the UK [39] considerably lower (7\%) prevalence was observed using microscopical methods on one unfixed stool specimen for diagnosis. The higher prevalence in our study can be due to selection of our hospital population, assuming pathogenicity of Blastocystis, but also to improved sensitivity of the diagnostic procedures used.

The high prevalence in our population was observed both by sequence-confirmed PCR and by microscopy. With excellent specificity of both methods, the prevalence observed most likely represents the true occurrence of the parasite in our population. The high sensitivity of microscopy was remarkable compared to previous studies $[32,39,40]$. This can be explained by the use of two fixed samples in the Triple Faeces Test rather than single or fresh (non-preserved) samples as used in previous studies [38,39]. In our study, microscopy on two stool samples, compared to one sample, increased the sensitivity of microscopy up to $10 \%$. This is in agreement with earlier reports where intermittent shedding of Blastocystis was observed and stresses the need for multiple stool examinations with microscopy before reporting a negative result $[41,42]$. Moreover, with the Triple Faeces Test patients themselves add stool samples immediately after defaecation to SAF fixative, reducing the time to fixation to a minimum. This is in contrast to earlier reports where stools were added to a fixative only after delivery in the laboratory [32]. In fresh samples, vegetative stages of protozoa, including Blastocystis, may rapidly deteriorate and become microscopically unrecognizable [43]. Timely fixation therefore is important for high diagnostic sensitivity of Blastocystis with microscopy.

Although microscopy on two samples proved very effective for diagnosis of Blastocystis, equal results with PCR diagnosis were obtained with examination of one sample only. In addition, determination of the subtype can be obtained, which is not possible by microscopy. We included sequence confirmation as a criterion for a positive PCR, to assure 100\% specificity. Sequence-confirmed PCR was positive in 103 out of 107 (97\%) positive patients. Discrepancies between sequence-confirmed PCR and microscopy in our study probably were related to very low numbers of Blastocystis shedded in the stools, resulting in insufficient amounts of PCR product to obtain a sequence.

The subtype distribution in the study population showed six different subtypes: ST1, ST2, ST3, ST4, ST6 and ST7. Subtypes 1-4 have been found as the most common subtypes in previous studies as well, [19,22,44-49], including molecular studies using samples initially cultured in vitro. Various studies have identified ST3 as the predominant subtype, as was true for our study population. Subtype 4 was relatively frequent in our study group (12\%) as compared to studies worldwide [50], although a more similar prevalence of this subtype was observed in studies from Europe [25]. The differences in relative abundance of the subtypes 1-4 suggests that differences in transmission efficiency may exist between them. These could include differences in cyst numbers, cyst survival, exposure to cysts, infectious dose or transmission cycles. The single subtype 6 and subtype 7 cases may reflect zoonotic infections, as these subtypes have been reported in stools from birds [51,52].

The significantly higher prevalence of Blastocystis infection in patients presenting at the Department of Tropical Medicine as compared to other departments, suggests that travel to tropical countries can increase the risk of Blastocystis infection, as has been observed in earlier studies $[7,8,53,54]$. High prevalence of Blastocystis has been reported in developing countries $[1,44,55,56]$. Despite the higher prevalence, the Blastocystis subtype distribution among travelers and patients of other clinical departments was similar. This suggests that general poor hygiene and sanitation in many of the tropical countries rather than specific exposure to certain Blastocystis subtypes, causes the observed increase in prevalence. Unfortunately, no specific data about previous travel to tropical countries were available for the patients from departments other than the Department of Tropical Medicine. 


\section{Conclusions}

In conclusion, fast and reliable diagnosis of Blastocystis infection in routine clinical parasitology is possible by advanced microscopy on multiple fixed stool samples with immediate fixation after production of stools. In terms of sensitivity, specificity and subtype determination, direct PCR on a single stool sample is also an excellent diagnostic tool. The prevalence of Blastocystis infection and the ongoing debate about pathogenicity warrant the use of good diagnostic methods in routine clinical laboratories. Other subjects of interest are patients with gastrointestinal complaints returning from the tropics without pathogenic microorganisms but only Blastocystis in stools. Further studies on pathogenicity, and especially the importance of subtypes of Blastocystis, are needed, as well as identification of an effective treatment. For many of these studies excellent laboratory diagnosis is a prerequisite.

\section{Competing interests}

The authors declare that they have no competing interests.

\section{Authors' contributions}

$A B$ designed the study, analyzed data and drafted the manuscript, EMSW-B and CJAW collected and analyzed advanced microscopy data, HG and NV collected and analyzed PCR and sequence data, MVV and AG collected patient data and helped in drafting the manuscript, TVG conceived, designed and coordinated the study and drafted the manuscript. All authors read and approved the final manuscript.

\section{Acknowledgements}

We thank Leny Nieuwendijk, Patricia Broekhuizen, Karin Prtenjača-Wolbers, Henk Hofwegen, Leonie van Boetzelaer, Bastiaan Spanjaard and Kiran Ramdin, for their excellent technical assistance to this study.

\section{Author details}

'Parasitology Section, Department of Medical Microbiology, Center for Infection and Immunity Amsterdam (CINIMA), Academic Medical Center, Amsterdam, The Netherlands. ${ }^{2}$ Center for Tropical and Travel Medicine, Department of Infectious Diseases, Academic Medical Center, University of Amsterdam, Amsterdam, The Netherlands. ${ }^{3}$ Infectious Diseases Epidemiological Unit, Public Health School, Université Libre de Bruxelles, Brussels, Belgium.

Received: 1 February 2013 Accepted: 19 August 2013

Published: 23 August 2013

\section{References}

1. Pegelow K, Gross R, Pietrzik K, Lukito W, Richards AL, Fryauff DJ: Parasitological and nutritional situation of school children in the Sukaraja district, West Java, Indonesia. Southeast Asian J Trop Med Public Health 1997, 28:173-190.

2. Horiki N, Maruyama M, Fujita Y, Yonekura T, Minato S, Kaneda Y: Epidemiologic survey of Blastocystis hominis infection in Japan. Am J Trop Med Hyg 1997, 56:370-374.

3. Stensvold CR, Nielsen HV, Molbak K, Smith HV: Pursuing the clinical significance of Blastocystis-diagnostic limitations. Trends Parasitol 2009, 25:23-29.

4. Li LH, Zhou XN, Du ZW, Wang XZ, Wang LB, Jiang JY, Yoshikawa H, Steinmann P, Utzinger J, Wu Z, Chen JX, Chen SH, Zhang L: Molecular epidemiology of human Blastocystis in a village in Yunnan province, China. Parasitol Int 2007, 56:281-286.

5. Eroglu F, Koltas IS: Evaluation of the transmission mode of B. hominis by using PCR method. Parasitol Res 2010, 107:841-845.

6. Leelayoova S, Siripattanapipong S, Thathaisong U, Naaglor T, Taamasri P, Piyaraj P, Mungthin M: Drinking water: a possible source of Blastocystis spp. subtype 1 infection in schoolchildren of a rural community in central Thailand. Am J Trop Med Hyg 2008, 79:401-406.

7. Sohail MR, Fischer PR: Blastocystis hominis and travelers. Travel Med Infect Dis 2005, 3:33-38.

8. Kain KC, Noble MA, Freeman HJ, Barteluk RL: Epidemiology and clinical features associated with Blastocystis hominis infection. Diagn Microbiol Infect Dis 1987, 8:235-244.

9. Yakoob J, Jafri W, Jafri N, Khan R, Islam M, Beg MA, Zaman V: Irritable bowel syndrome: in search of an etiology: role of Blastocystis hominis. Am J Trop Med Hyg 2004, 70:383-385.

10. Boorom KF, Smith H, Nimri L, Viscogliosi E, Spanakos G, Parkar U, Li LH, Zhou XN, Ok UZ, Leelayoova S, Jones MS: Oh my aching gut: irritable bowel syndrome, Blastocystis, and asymptomatic infection. Parasit Vectors 2008, 1:40.

11. Poirier P, Wawrzyniak I, Vivares CP, Delbac F, El AH: New insights into Blastocystis spp.: a potential link with irritable bowel syndrome. PLoS Pathog 2012, 8: 1002545.

12. Yakoob J, Jafri W, Beg MA, Abbas Z, Naz S, Islam M, Khan R: Irritable bowel syndrome: is it associated with genotypes of Blastocystis hominis. Parasitol Res 2010, 106:1033-1038

13. Dogruman-Al F, Simsek Z, Boorom K, Ekici E, Sahin M, Tuncer C, Kustimur S, Altinbas A: Comparison of methods for detection of Blastocystis infection in routinely submitted stool samples, and also in IBS/IBD Patients in Ankara. Turkey PLoS One 2010, 5:e15484.

14. Gassama A, Sow PS, Fall F, Camara P, Gueye N'diaye A, Seng R, Samb B, M'Boup S, Idara Kane A: Ordinary and opportunistic enteropathogens associated with diarrhea in Senegalese adults in relation to human immunodeficiency virus serostatus. Int I Infect Dis 2001, 5:192-198.

15. Tan TC, Ong SC, Suresh KG: Genetic variability of Blastocystis sp. isolates obtained from cancer and HIV/AIDS patients. Parasitol Res 2009, 105:1283-1286

16. Leder K, Hellard ME, Sinclair MI, Fairley CK, Wolfe R: No correlation between clinical symptoms and Blastocystis hominis in immunocompetent individuals. J Gastroenterol Hepatol 2005, 20:1390-1394.

17. Udkow MP, Markell EK: Blastocystis hominis: prevalence in asymptomatic versus symptomatic hosts. J Infect Dis 1993, 168:242-244.

18. Sun T, Katz S, Tanenbaum B, Schenone C: Questionable clinical significance of Blastocystis hominis infection. Am J Gastroenterol 1989, 84:1543-1547

19. Dogruman AF, Dagci H, Yoshikawa H, Kurt O, Demirel M: A possible link between subtype 2 and asymptomatic infections of Blastocystis hominis. Parasitol Res 2008, 103:685-689.

20. Dominguez-Marquez MV, Guna R, Munoz C, Gomez-Munoz MT, Borras R: High prevalence of subtype 4 among isolates of Blastocystis hominis from symptomatic patients of a health district of Valencia (Spain). Parasitol Res 2009, 105:949-955.

21. Stensvold CR, Suresh GK, Tan KS, Thompson RC, Traub RJ, Viscogliosi E, Yoshikawa H, Clark CG: Terminology for Blastocystis subtypes-a consensus. Trends Parasitol 2007, 23:93-96.

22. Stensvold CR, Lewis HC, Hammerum AM, Porsbo LJ, Nielsen SS, Olsen KE, Arendrup MC, Nielsen HV, Molbak K: Blastocystis: unravelling potential risk factors and clinical significance of a common but neglected parasite. Epidemiol Infect 2009, 137:1655-1663.

23. Tan TC, Suresh KG, Smith HV: Phenotypic and genotypic characterisation of Blastocystis hominis isolates implicates subtype 3 as a subtype with pathogenic potential. Parasitol Res 2008, 104:85-93.

24. Alfellani MA, Stensvold CR, Vidal-Lapiedra A, Onuoha ES, Fagbenro-Beyioku AF, Clark CG: Variable geographic distribution of Blastocystis subtypes and its potential implications. Acta Trop 2013, 126:11-18.

25. Forsell J, Granlund M, Stensvold CR, Clark CG, Evengard B: Subtype analysis of Blastocystis isolates in Swedish patients. Eur J Clin Microbiol Infect Dis 2012, 31:1689-1696.

26. Poirier P, Wawrzyniak I, Albert A, El AH, Delbac F, Livrelli V: Development and evaluation of a real-time PCR assay for detection and quantification of blastocystis parasites in human stool samples: prospective study of patients with hematological malignancies. J Clin Microbiol 2011, 49:975-983.

27. Olivo-Diaz A, Romero-Valdovinos M, Gudino-Ramirez A, Reyes-Gordillo J, Jimenez-Gonzalez DE, Ramirez-Miranda ME, Martinez-Flores WA, MartinezHernandez F, Flisser A, Maravilla P: Findings related to IL-8 and IL-10 gene 
polymorphisms in a Mexican patient population with irritable bowel syndrome infected with Blastocystis. Parasitol Res 2012, 111:487-491.

28. Stensvold CR, Alfellani MA, Norskov-Lauritsen S, Prip K, Victory EL, Maddox C, Nielsen HV, Clark CG: Subtype distribution of Blastocystis isolates from synanthropic and zoo animals and identification of a new subtype. Int J Parasitol 2009, 39:473-479.

29. Tan KS: New insights on classification, identification, and clinical relevance of Blastocystis spp. Clin Microbiol Rev 2008, 21:639-665.

30. Parkar U, Traub RJ, Vitali S, Elliot A, Levecke B, Robertson I, Geurden T, Steele J, Drake B, Thompson RC: Molecular characterization of Blastocystis isolates from zoo animals and their animal-keepers. Vet Parasitol 2010, 169:8-17.

31. Alfellani MA, Taner-Mulla D, Jacob AS, Imeede CA, Yoshikawa H, Stensvold CR, Clark CG: Genetic diversity of blastocystis in livestock and zoo animals. Protist 2013, 164:497-509.

32. Roberts T, Stark D, Harkness J, Ellis J: Subtype distribution of Blastocystis isolates identified in a Sydney population and pathogenic potential of Blastocystis. Eur J Clin Microbiol Infect Dis 2012, 32:335-343.

33. Stensvold CR, Arendrup MC, Jespersgaard C, Molbak K, Nielsen HV Detecting Blastocystis using parasitologic and DNA-based methods: a comparative study. Diagn Microbiol Infect Dis 2007, 59:303-307.

34. Stensvold CR, Ahmed UN, Andersen LO, Nielsen HV: Development and evaluation of a genus-specific, probe-based, internal-process-controlled real-time PCR assay for sensitive and specific detection of Blastocystis spp. J Clin Microbiol 2012, 50:1847-1851.

35. Van Gool T, Weijts R, Lommerse E, Mank TG: Triple faeces test: an effective tool for detection of intestinal parasites in routine clinical practice. Eur J Clin Microbiol Infect Dis 2003, 22:284-290.

36. Niesters HG: Quantitation of viral load using real-time amplification techniques. Methods 2001, 25:419-429.

37. Tamura K, Dudley J, Nei M, Kumar S: MEGA4: Molecular Evolutionary Genetics Analysis (MEGA) software version 4.0. Mol Biol Evol 2007, 24:1596-1599.

38. Masucci L, Graffeo R, Bani S, Bugli F, Boccia S, Nicolotti N, Fiori B, Fadda G, Spanu T: Intestinal parasites isolated in a large teaching hospital, Italy. Euro Surveill 2011, 16:19891.

39. Windsor JJ, Macfarlane L, Hughes-Thapa G, Jones SK, Whiteside TM: Incidence of Blastocystis hominis in faecal samples submitted for routine microbiological analysis. Br J Biomed Sci 2002, 59:154-157.

40. Zaman $\mathrm{V}$, Khan $\mathrm{KZ}$ : A comparison of direct microscopy with culture for the diagnosis of Blastocystis hominis. Southeast Asian J Trop Med Public Health 1994, 25:792-793.

41. Vennila GD, Suresh KG, Khairul AA, Rajah S, Saminathan R, Sivanandan S, Ramakrishnan K: Irregular shedding of Blastocystis hominis. Parasitol Res 1999, 85:162-164.

42. Suresh K, Venilla GD, Tan TC, Rohela M: In vivo encystation of Blastocystis hominis. Parasitol Res 2009, 104:1373-1380.

43. Mank TG, Zaat JO, Blotkamp J, Polderman AM: Comparison of fresh versus sodium acetate acetic acid formalin preserved stool specimens for diagnosis of intestinal protozoal infections. Eur J Clin Microbiol Infect Dis 1995, 14:1076-1081.

44. Li LH, Zhang XP, Lv S, Zhang L, Yoshikawa H, Wu Z, Steinmann P, Utzinger J, Tong XM, Chen SH, Zhou XN: Cross-sectional surveys and subtype classification of human Blastocystis isolates from four epidemiological settings in China. Parasitol Res 2007, 102:83-90.

45. Scicluna SM, Tawari B, Clark CG: DNA barcoding of blastocystis. Protist 2006, 157:77-85.

46. Stensvold CR, Traub RJ, von Samson-Himmelstjerna G, Jespersgaard C, Nielsen HV, Thompson RC: Blastocystis: subtyping isolates using pyrosequencing technology. Exp Parasitol 2007, 116:111-119.

47. Stensvold R, Brillowska-Dabrowska A, Nielsen HV, Arendrup MC: Detection of Blastocystis hominis in unpreserved stool specimens by using polymerase chain reaction. J Parasitol 2006, 92:1081-1087

48. Yoshikawa H, Wu Z, Kimata I, Iseki M, Ali IK, Hossain MB, Zaman V, Haque R, Takahashi Y: Polymerase chain reaction-based genotype classification among human Blastocystis hominis populations isolated from different countries. Parasitol Res 2004, 92:22-29.

49. Yan Y, Su S, Lai R, Liao H, Ye J, Li X, Luo X, Chen G: Genetic variability of Blastocystis hominis isolates in China. Parasitol Res 2006, 99:597-601.
50. Souppart L, Sanciu G, Cian A, Wawrzyniak I, Delbac F, Capron M, Dei-Cas E, Boorom K, Delhaes L, Viscogliosi E: Molecular epidemiology of human Blastocystis isolates in France. Parasitol Res 2009, 105:413-421.

51. Noel C, Dufernez F, Gerbod D, Edgcomb VP, Gado Viscogliosi P, Ho LC, Singh M, Wintjens R, Sogin ML, Capron M, Pierce R, Zenner L, Viscogliosi E: Molecular phylogenies of Blastocystis isolates from different hosts: implications for genetic diversity, identification of species, and zoonosis. J Clin Microbiol 2005, 43:348-355.

52. Yoshikawa H, Abe N, Wu Z: PCR-based identification of zoonotic isolates of Blastocystis from mammals and birds. Microbiology 2004, 150:1147-1151.

53. Jelinek T, Peyerl G, Loscher T, Von SF, Nothdurft HD: The role of Blastocystis hominis as a possible intestinal pathogen in travellers. J Infect 1997, 35:63-66

54. Shlim DR, Hoge CW, Rajah R, Rabold JG, Echeverria P: Is Blastocystis hominis a cause of diarrhea in travelers? A prospective controlled study in Nepal. Clin Infect Dis 1995, 21:97-101.

55. Graczyk TK, Shiff CK, Tamang L, Munsaka F, Beitin AM, Moss WJ: The association of Blastocystis hominis and Endolimax nana with diarrheal stools in Zambian school-age children. Parasitol Res 2005, 98:38-43.

56. Noor Azian MY, San YM, Gan CC, Yusri MY, Nurulsyamzawaty Y, Zuhaizam $\mathrm{AH}$, Maslawaty MN, Norparina I, Vythilingam I: Prevalence of intestinal protozoa in an aborigine community in Pahang, Malaysia. Trop Biomed 2007, 24:55-62.

doi:10.1186/1471-2334-13-389

Cite this article as: Bart et al:: Diagnosis and subtype analysis of Blastocystis sp. in 442 patients in a hospital setting in the Netherlands. BMC Infectious Diseases 2013 13:389.

\section{Submit your next manuscript to BioMed Central and take full advantage of:}

- Convenient online submission

- Thorough peer review

- No space constraints or color figure charges

- Immediate publication on acceptance

- Inclusion in PubMed, CAS, Scopus and Google Scholar

- Research which is freely available for redistribution 